\title{
Análisis comparativo de mezclas suelo cemento modificadas con materiales no biodegradables. Metodología de la portland cement association
}

\section{Comparative analysis of cement modified soil mixes with non-biodegradable materials. Portland cement association methodology}

\section{Sandra Elodia Ospina Lozano}

Ingeniera civil, Magister en ingeniería

Facultad de ingeniería civil, Universidad de La Salle

Bogotá, (Colombia).

seospina@unisalle.edu.co

\section{Adriana María López Velandia}

Estudiante ingeniería civil, Semillero de investigación INDETEC

Facultad de ingeniería civil, Universidad de La Salle

Bogotá, (Colombia).

ladriana87@unisalle.edu.co

\author{
Camilo Andrés Nieves Castro \\ Estudiante ingeniería civil, Semillero de investigación INDETEC \\ Facultad de ingeniería civil, Universidad de La Salle \\ Bogotá, (Colombia). \\ cnieves35@unisalle.edu.co
}

Fecha de recepción: 24 febrero de 2017 / Fecha de aprobación: 19 de junio de 2017

\section{RESUMEN}

Este artículo presenta el análisis comparativo de investigaciones realizadas en la Universidad de La Salle - Colombia desde el año 2012, referente a mezclas suelo cemento modificadas diseñadas con la metodología de la Portland Cement Association [PCA] (ACI, 1997), y guiadas por el artículo 350 del Instituto Nacional de Vías [Invías] del año 2013, que refiere a las características que debe cumplir una mezcla granular mejorada con cemento para control de calidad en obra, de aplicación nacional en Colombia. Inicialmente se recopila y organiza la información de manera que se identifiquen criterios comparativos de los resultados obtenidos de mezclas suelo cemento modificadas con materiales no biodegradables, como vidrio templado, poliestireno expandido [EPS], tereftalato de polietileno [PET], polietileno de alta densidad [PEHD] y grano de caucho. Enseguida se evaluaron los resultados obtenidos de dicho análisis comparativo, cuyas variaciones en los contenidos óptimos de cemento discrepan de los rangos sugeridos de la PCA, lo que lleva a plantear una discusión sobre la aplicación de esta metodología para mezclas suelo cemento modificadas, proponiendo nuevos rangos de parámetros de control para futuras investigaciones, los cuales servirán como punto de partida para proponer una nueva metodología de diseño en mezclas suelo cemento empleando dichos materiales. Sin embargo, el análisis aquí mostrado evidencia la necesidad de enriquecer con un mayor número de datos las recomendaciones que se presentan, para dar validez soportado en un procesamiento estadístico suficiente.

PALABRAS CLAVES: Mezclas suelo cemento modificadas, metodología PCA, materiales no biodegradables, Porcentaje óptimo de cemento.

\section{ABSTRACT}

This article presents the comparative analysis of research carried out at the La Salle University - Colombia since the year 2012, regarding modified cement mixtures designed with the Portland Cement Association (PCA) methodology (ACI, 1997) and guided by the Article 350 of the National Institute of Roads [Invias] of the year 2013, which refers to the characteristics that must be met by an improved granular mixture with cement for quality control on site, of national application in Colombia. Initially, information is collected and organized to identify comparative criteria for the results obtained from cement-based mixtures modified with non-biodegradable materials such as tempered glass, expanded polystyrene [EPS], polyethylene terephthalate [PET], high density polyethylene [PEHD] and rubber grain. The results obtained from this comparative analysis were then evaluated, whose variations in the optimal cement contents differ from the suggested ranges of the PCA, which leads to a discussion on the application of this methodology for modified cement mixtures, proposing new ranges Of control parameters for future research, which will serve as a starting point for proposing a new design methodology in cement soil mixtures using such materials. However, the analysis presented here evidences the need to enrich the recommendations that are presented with a greater number of data, to give validity supported in sufficient statistical processing.

KEY WORDS: Modified ground cement mixtures, methodology PCA, non-biodegradable materials, optimal percentage of cement. 


\section{INTRODUCCIÓN}

El presente artículo corresponde a una publicación de revisión; su objetivo es el de plantear una discusión referente a los rangos recomendados en la metodología PCA para la adición de cemento, a las mezclas suelo cemento en las cuales se incorporaron materiales no biodegradables como vidrio templado, PEHD, PET, EPS y grano de caucho.
Los materiales que se trabajaron en las investigaciones previas de mezclas suelo cemento modificadas, corresponden a aquellos que han tenido mayor impacto en el medio ambiente, debido a la contaminación que generan, tiempo de descomposición y el volumen que ocupan sus desechos (Cuadro 1). "En Colombia de las 11.6 millones de toneladas de residuos que se generan al año, solo se aprovecha un $17 \%$, caso contrario a países como Holanda y Alemania donde se aprovechan el $99 \%$ y $98 \%$ de los residuos respectivamente" (Rojas, 2016).

Cuadro 1. Características de los materiales no biodegradables empleados para modificar mezclas suelo cemento.

\begin{tabular}{|c|c|c|c|c|}
\hline Material & Definición & $\begin{array}{l}\text { Volumen } \\
\text { generado por } \\
\text { año (Ton) }\end{array}$ & $\begin{array}{c}\text { Tiempo de descomposición } \\
\text { años }\end{array}$ & Dato de interés \\
\hline $\begin{array}{l}\text { Grano de } \\
\text { caucho }\end{array}$ & $\begin{array}{l}\text { Se obtiene a través de un proceso que se } \\
\text { utiliza por trituración mecánica y sepa- } \\
\text { ración de los materiales que conforman } \\
\text { las Ilantas. Es un producto no tóxico, de } \\
\text { forma granular y suave olor que caracte- } \\
\text { riza al caucho. (Ramírez. N, 2006) }\end{array}$ & $\begin{array}{l}61000 \\
\text { (Ministerio } \\
\text { de Ambiente- } \\
\text { Colombia, } \\
\text { 2010) }\end{array}$ & 600 & $\begin{array}{l}\text { Para el } 2008 \text { en Colombia se consumían } \\
\text { 4'493.092 llantas, solamente } 5 \% \text { de ellas es } \\
\text { reciclado o renovado, el } 2 \% \text { es utilizado para } \\
\text { generar energía y otro } 2 \% \text { son utilizadas en } \\
\text { artesanías, lo que deja un } 91 \% \text { de llantas que se } \\
\text { quedan como basura. (Revista Dinero, 2009). }\end{array}$ \\
\hline $\begin{array}{l}\text { Tereftalato de } \\
\text { polietileno }\end{array}$ & $\begin{array}{l}\text { Es un tipo de plástico muy usado en en- } \\
\text { vases de bebidas y textiles. El PET en ge- } \\
\text { neral se caracteriza por su elevada pureza, } \\
\text { alta resistencia y tenacidad. De acuerdo } \\
\text { con su orientación presenta propiedades } \\
\text { de transparencia y resistencia química. } \\
\text { (Tecnología de los plásticos, 2011). }\end{array}$ & 60000 & 100 a 1.000 & $\begin{array}{l}\text { Las aplicaciones más conocidas del tereftalato } \\
\text { de polietileno están en cajas para bebidas, } \\
\text { donde su vida útil es de } 5 \text { a } 7 \text { años aproxima- } \\
\text { damente, bolsas plásticas con vida útil menor } \\
\text { a un año y películas de invernadero con vida } \\
\text { útil de } 2 \text { a } 3 \text { años. } \\
\text { (Ministerio de Ambiente-Colombia, 2004). }\end{array}$ \\
\hline $\begin{array}{l}\text { Poliestireno } \\
\text { expandido }\end{array}$ & $\begin{array}{l}\text { Es un material plástico espumado, } \\
\text { derivado del poliestireno y utilizado en } \\
\text { el sector del envase y la construcción. } \\
\text { (ANAPE, 2017) }\end{array}$ & 6000 & 500 a 1.000 & $\begin{array}{l}\text { Los residuos de poliestireno en los rellenos sani- } \\
\text { tarios ocupan un espacio bastante significativo } \\
\text { ya que el volumen equivalente a una tonelada } \\
\text { de poliestireno es el mismo para } 100 \text { toneladas } \\
\text { de otro tipo de materiales. (Junca. I, 2014). }\end{array}$ \\
\hline $\begin{array}{l}\text { Polietileno de } \\
\text { alta densidad }\end{array}$ & $\begin{array}{l}\text { El polietileno de alta densidad es un } \\
\text { polímero resultado de la polimerización } \\
\text { del etileno. Es posiblemente el plástico } \\
\text { más popular del mundo. } \\
\text { (M\&M Plásticos, 2012). }\end{array}$ & 75000 & 500 & $\begin{array}{l}\text { El peso promedio de una botella de dos litros } \\
\text { ha pasado de } 120 \text { gramos en la década del } \\
70 \text { a } 67 \text { gramos en la década del } 90 \text {. De los } \\
\text { desechos que llegan al relleno sanitario Doña } \\
\text { Juana (Bogotá) 5,8\% corresponden a residuos } \\
\text { de PEHD. (Álzate S. \& Tafur F. 2006). En } 2015 \text { en } \\
\text { Colombia se recuperaron entre } 3.000 \text { y } 3.500 \\
\text { toneladas de envases PEHD, lo que representa } \\
\text { tan solo } 26 \% \text { del total. (Zarta D. 2016) }\end{array}$ \\
\hline
\end{tabular}

Se efectuó el estudio comparativo de los resultados para las diferentes mezclas a través de la revisión de las fuentes bibliográficas y trabajos de investigación. Bajo esta perspectiva, se realizó un análisis de las propiedades y características de estas mezclas, las cuales cumplen con los requerimientos establecidos en la Tabla 350-2 del artículo 350 del Invías, y en la metodología
PCA (ACI, 1997). Sin embargo, aunque se lograron alcanzar las resistencias y durabilidades deseadas, en algunos casos estos valores estuvieron fuera de los rangos sugeridos de adición de cemento según la metodología PCA, esto plantea una necesidad de realizar una adaptación de la metodología para permitir la innovación e incorporación de nuevos materiales. 
El método de diseño para mezclas suelo cemento PCA, evalúa el comportamiento de esta mezcla, cuyo principal requisito es resistir las variaciones de las condiciones climáticas. De este modo, el elemento básico de una mezcla suelo - cemento, es la cantidad de cemento requerida para producir una mezcla que resista los esfuerzos producidos en los ensayos de durabilidad por humedecimiento y secado.

\section{METODOLOGÍA}

Se realizó una recopilación bibliográfica de las investigaciones en mezclas suelo cemento modificadas con materiales no biodegradables (Acero y Mogollón, 2015), (Buitrago y González, 2015), (Cruz y Díaz, 2015), (Infante, 2015), (Meneses y Fuentes, 2015), (Ochoa y Valderrama, 2012), (Sarmiento y Bustos, 2016), (Velandia y Parra, 2016), que fue organizada de acuerdo al tipo de suelo y al método de modificación (adición o sustitución) según se presenta en el cuadro 2.

Cuadro 2. Características de composición de las mezclas suelo cemento modificadas

\begin{tabular}{|c|c|c|c|c|c|c|c|c|}
\hline \multicolumn{2}{|c|}{ Clasificación } & \multirow{2}{*}{$\begin{array}{l}\text { Material no } \\
\text { biodegradable }\end{array}$} & \multirow{2}{*}{$\begin{array}{l}\text { Nombre asignado } \\
\text { a la mezcla }\end{array}$} & \multicolumn{2}{|c|}{ Método de modificación } & \multirow{2}{*}{$\begin{array}{l}\% \text { material no biodegradable } \\
\text { (sustituye } 0 \text { adiciona) }\end{array}$} & \multicolumn{2}{|c|}{ Franja granulométrica } \\
\hline AASTHO & USCS & & & Sustitución & Adición & & Pasa tamiz & Retiene tamiz \\
\hline A-1-a & GW & PET & PT2 & $\ddot{u}$ & $x$ & $0-25-50-75-100$ & $N^{\circ} 4$ & $N^{\circ} 16$ \\
\hline$A-2-4$ & SM & Grano de caucho & $\mathrm{C} 1$ & $\ddot{u}$ & $x$ & $0-25-50-75-100$ & $N^{\circ} 30$ & $N^{\circ} 80$ \\
\hline$A-2-4$ & SM & Grano de caucho & $\mathrm{C} 2$ & ü & $x$ & $0-25-50-75-100$ & $N^{\circ} 8$ & $N^{\circ} 16$ \\
\hline A-2-4 & SM & PET & PT1 & $x$ & ü & $0-5-10-15$ & \multicolumn{2}{|c|}{ No aplica, adición en peso. } \\
\hline$A-2-4$ & SM & Vidrio templado & V & $x$ & $\ddot{u}$ & $0-5-10-15$ & \multicolumn{2}{|c|}{ No aplica, adición en peso. } \\
\hline$A-2-6$ & SM & EPS & $P$ & $\ddot{u}$ & $x$ & $0-12.5-25-37.5-50$ & $\mathrm{~N}^{\circ} 10$ & $N^{\circ} 20$ \\
\hline A-2-6 & SM & PEHD & PD & $\ddot{u}$ & $x$ & $0-25-50-75-100$ & $N^{\circ} 4$ & $\mathrm{~N}^{\circ} 10$ \\
\hline$A-4$ & $\mathrm{CL}$ & Grano de caucho & $\mathrm{C} 3$ & $x$ & $\ddot{u}$ & $0-5-25-50$ & \multicolumn{2}{|c|}{ No aplica, adición en peso. } \\
\hline
\end{tabular}

Nota: $\checkmark$ Se realizó, $\times$ No se realizó.

Los procesos de adición y sustitución en las mezclas fueron hechos siempre por peso exceptuando el del EPS, el cual fue realizado por volumen.

Los materiales granulares patrón (primera columna del cuadro 2), y las mezclas fueron evaluadas bajo los requerimientos del artículo 350-13 del Invías, conformando un grupo de ensayos presentados en el cuadro 3; los ensayos enlistados no fueron efectuados a todas las mezclas, pues el objeto de cada trabajo de investigación era variable.

Cuadro 3. Ensayos realizados a los materiales granulares patrón y las mezclas

\begin{tabular}{|c|c|c|}
\hline $\begin{array}{l}\text { Código de la } \\
\text { norma }\end{array}$ & Nombre del ensayo & Valor requerido \\
\hline INV E $121-13$ & Determinación del contenido orgánico de un suelo mediante el ensayo de pérdida por ignición & 1\% máx. \\
\hline INVE $122-13$ & Determinación en laboratorio del contenido de agua(humedad) de muestras de suelo, roca y mezclas de suelo- agregado & - \\
\hline INVE $123-13$ & Determinación de los tamaños de las partículas de los suelos & - \\
\hline INVE $125-13$ & Determinación del límite liquido de los suelos & - \\
\hline INVE $126-13$ & Límite plástico e índice de plasticidad de los suelos & - \\
\hline INVE $133-13$ & Equivalente de arena de suelos y agregados finos & 30\% Mín. \\
\hline INV E $142-13$ & Relaciones de humedad - peso unitario seco en los suelos (ensayo modificado de compactación) & N/A \\
\hline INV E $148-13$ & CBR de suelos compactados en el laboratorio y sobre muestra inalterada & 60\% Mín. \\
\hline INVE $211-13$ & Determinación terrones de arcilla y partículas deleznables en los agregados & 2\% Máx. \\
\hline \multirow{2}{*}{ INV E $218-13$} & \multirow{2}{*}{ Resistencia a la degradación de los agregados de tamaños menores de $37.5 \mathrm{~mm}\left(1 \frac{1}{1} 2^{\prime \prime}\right)$ por medio de la máquina de los ángeles } & 500 Rev.: 40\%Máx. \\
\hline & & 100 Rev.: 8\%Máx. \\
\hline INV E $220-13$ & Solidez de los agregados frente a la acción de soluciones de sulfato de sodio o magnesio & 18\% Máx. \\
\hline INV E $227-13$ & Porcentaje de partículas fracturadas en un agregado grueso & 50\% Mín. \\
\hline INV E $230-13$ & Índices de alargamiento y aplanamiento de los agregados para carreteras & - \\
\hline INV E $235-13$ & Valor de azul de metileno en agregados finos & 10 Máx. \\
\hline INVE $238-13$ & Determinación de la resistencia del agregado grueso a la degradación por abrasión utilizando el aparato micro-deval & 30\% Máx. \\
\hline
\end{tabular}


Las mezclas suelo cemento con material no biodegradable, fueron diseñadas según las dosificaciones de cemento estipuladas por la PCA (Cuadro 4).

De acuerdo con la organización de los datos se iniciaron las comparaciones mostrando las variaciones de las propiedades de resistencia a la compresión, porcentaje de cemento óptimo, durabilidad, humedad óptima y masa unitaria seca, estas comparaciones se muestran a través de figuras, donde se evidencia que algunas mezclas pueden ser más factibles de implementar como materiales de construcción.

Cuadro 4. Metodología de diseño PCA para mezclas suelo cemento. (ACl, 1997).

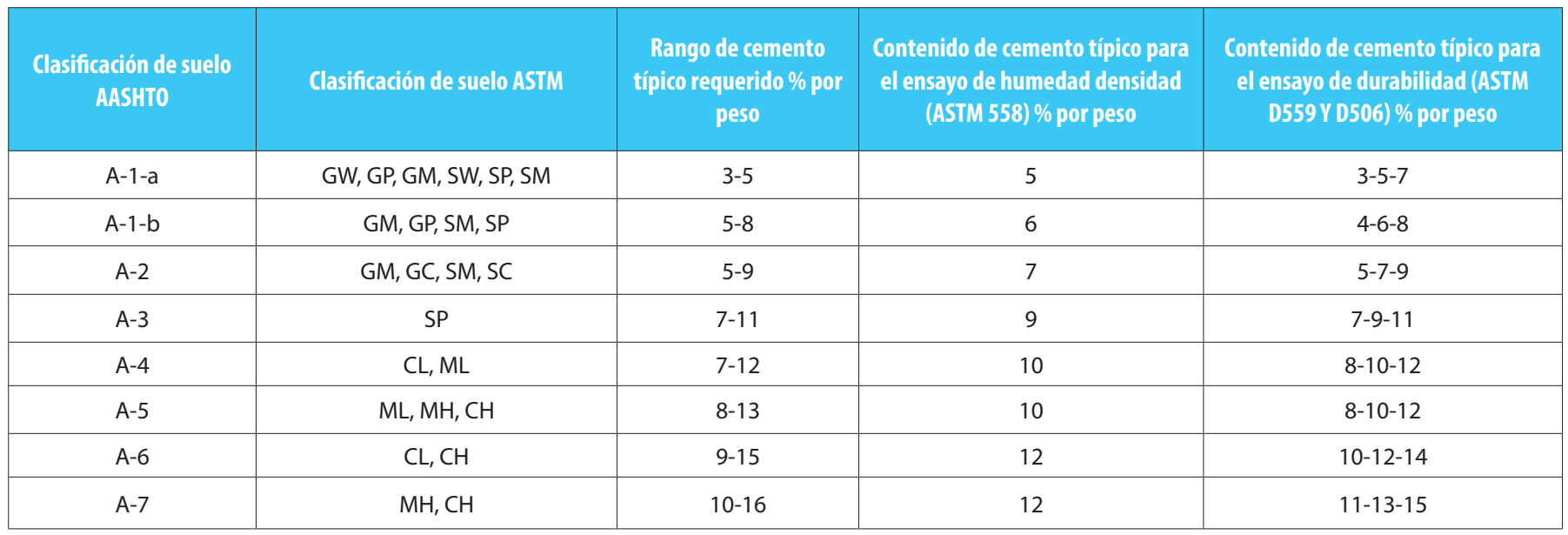

A continuación, se grafican los resultados obtenidos de las probetas con mayor porcentaje de cemento (valor máximo sugerido por la PCA y por ende mayor resistencia a la compresión), comparándolos con el requerimiento del artículo
350 del Invías (Figura 1), mostrando que para el porcentaje de cemento sugerido hay mezclas que no alcanzan la resistencia solicitada en el artículo.
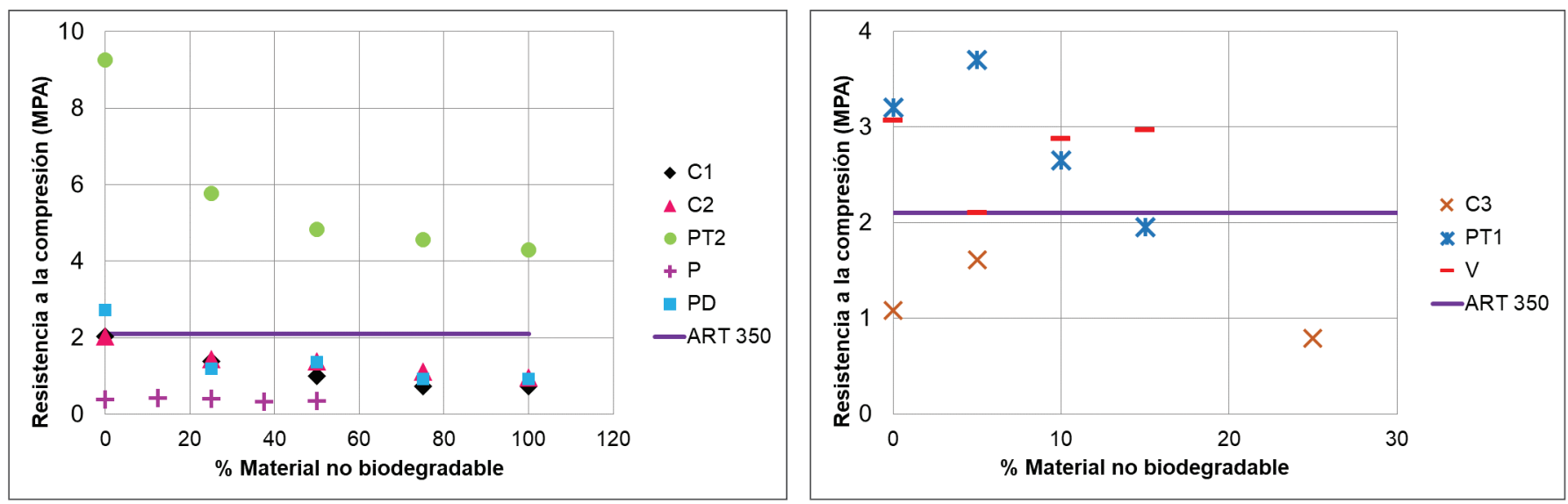

Figura 1. Comparación resistencia a la compresión suelos. a) por sustitución. b) por adición.

Según los parámetros establecidos por la PCA y los valores requeridos por el artículo 350 del Invías 2013, las muestras trabajadas con materiales como C1, C2, C3 y P no cumplen con la resistencia mínima de $2.1 \mathrm{MPa}$, caso contrario a lo que ocurre en las muestras trabajadas con PT1, PT2 y V, que cumplen con los valores mínimos establecidos para la resistencia a la compresión en todas las mezclas trabajadas, probablemente en la mezcla PT2 el cumplimiento del requerimiento obedezca a las características del material granular, que corresponde con una grava bien gradada. 
Una vez analizada la resistencia a la compresión, se define el porcentaje óptimo de cemento que alcance la resistencia mínima, según lo requerido en el artículo 350; cuando no se alcanzaba esta resistencia dentro del rango analizado de los porcentajes de cemento adicionados a las mezclas según las indicaciones de la PCA, fue necesaria la extrapolación de datos para obtener el contenido óptimo de cemento de cada mezcla. A continuación, se presenta la comparación de los diferentes tipos de suelos utilizados (Figuras 2 a 4) versus el rango de cemento utilizado para cada caso presentado en el cuadro 4.

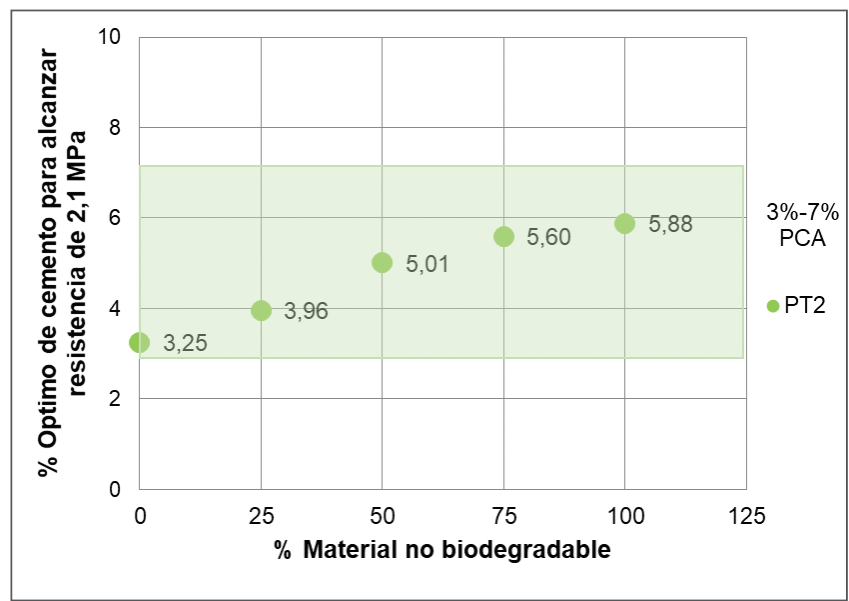

Figura 2. Porcentaje óptimo de cemento, suelo A-1-a por sustitución.
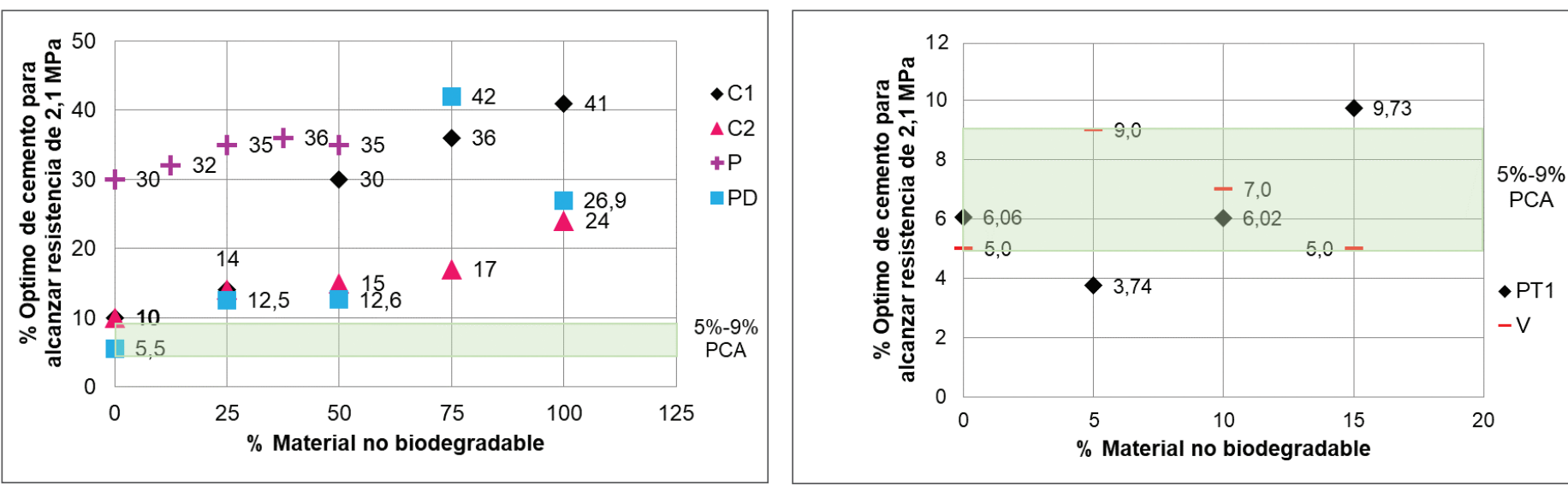

Figura 3. Porcentaje cemento óptimo, suelo A-2. a) por sustitución. b) por adición

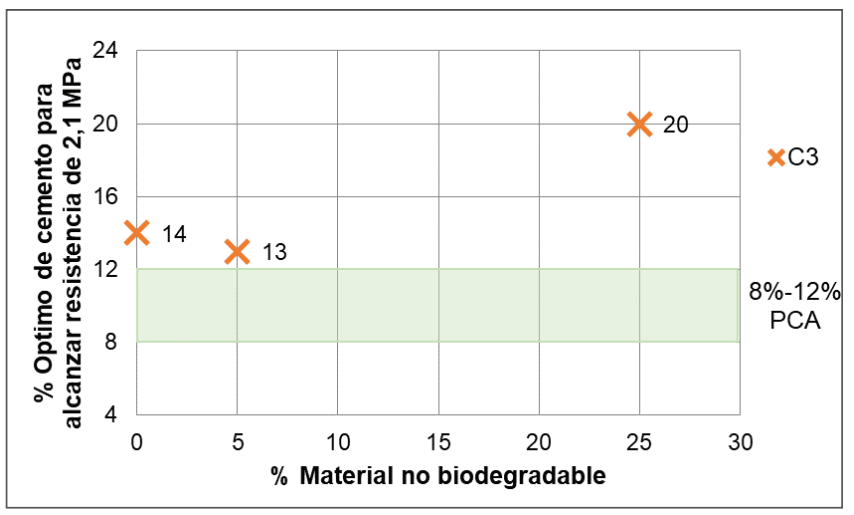

Figura 4. Porcentaje cemento óptimo, suelo A-4 por adición.
Se puede notar que el suelo A-1-a por sustitución, y A-2 por adición (para V, y parcialmente para PT1) alcanzan la resistencia mínima requerida dentro del rango del contenido de cemento sugerido por la PCA. Analizando el comportamiento de las adiciones para el suelo A-2, respecto al diseño de referencia ( $0 \%$ de material no biodegradable) se observa un comportamiento lineal considerando los porcentajes de adición utilizados de los modificadores V y PT1 (5\%, 10\% y 15\%), lo que permite observar que para valores que para el primer modificador $\mathrm{V}$ para porcentajes entre el $5 \%$ y el $15 \%$ de adición coincidiría con el rango de la PCA, mientras que para el modificador PT1 esto se cumpliría para el rango entre el $7 \%$ y el $14 \%$. 
Para el suelo A-2 por sustitución y A-4 por adición, es necesaria la extrapolación para obtener el contenido óptimo de cemento para lograr la misma resistencia.

Otra de las propiedades evaluadas, fue la de durabilidad o máxima pérdida de masa de la mezcla compactada en la prueba de humedecimiento y secado (Figura 5), según lo permitido en el artículo 350 para cada tipo de suelo.

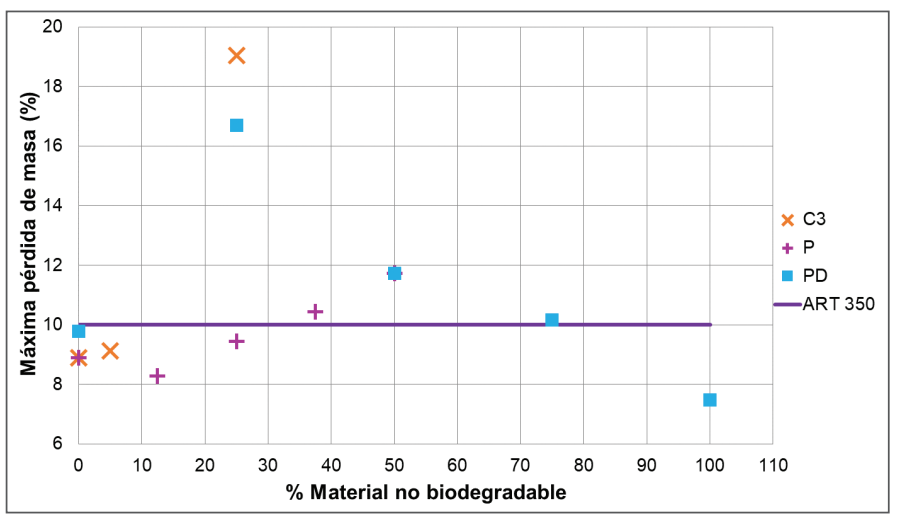

Figura 5. Máxima pérdida de masa de la mezcla compactada en prueba de humedecimiento y secado. a) Suelos A-2-6 y A-4. b) Suelos A-1-a y A-2-4

El ensayo de compactación es uno de los más importantes procedimientos de estudio y control de calidad de un terreno, en los trabajos analizados en este documento emplearon el ensayo de compactación Proctor Standard. A través de él es posible determinar la densidad seca máxima de un terreno en relación con su grado de humedad, a una energía de compactación
Según la Figura 5, los suelos de mejor calidad (Figura 5b) al ser modificados cumplen con el máximo de pérdida permitido según el artículo 350 (línea púrpura), pero en el caso de suelos arenolimosos o limoarcillosos, la pérdida presentada con la modificación es mayor que la máxima permitida, en este caso se debe observar que el material natural sin modificación estabilizado con cemento ( $0 \%$ material no biodegradable) presenta una pérdida muy cercana al límite máximo (Figura 5a).

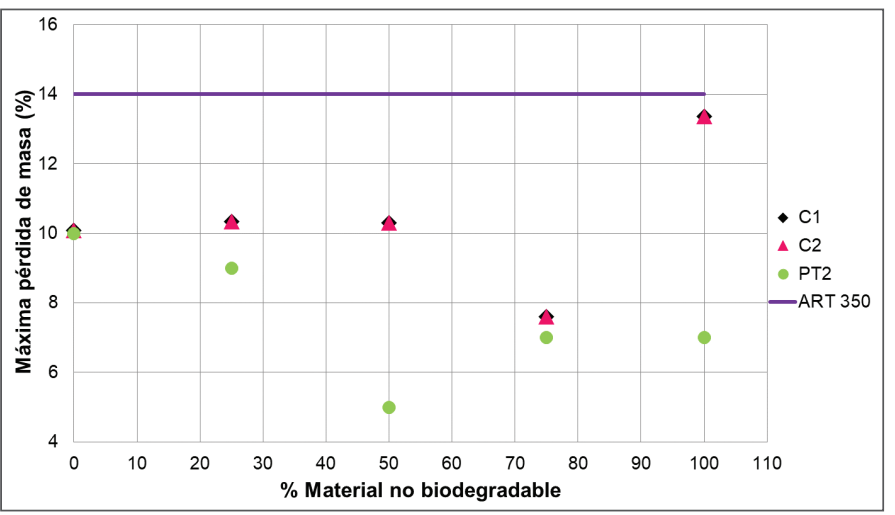

determinada (Chamba, 2014), la comparación del porcentaje de humedad óptima para las mezclas evaluadas se encuentra en la Figura 6, a su vez los resultados y comparación de la masa unitaria se encuentran en la Figura 7.

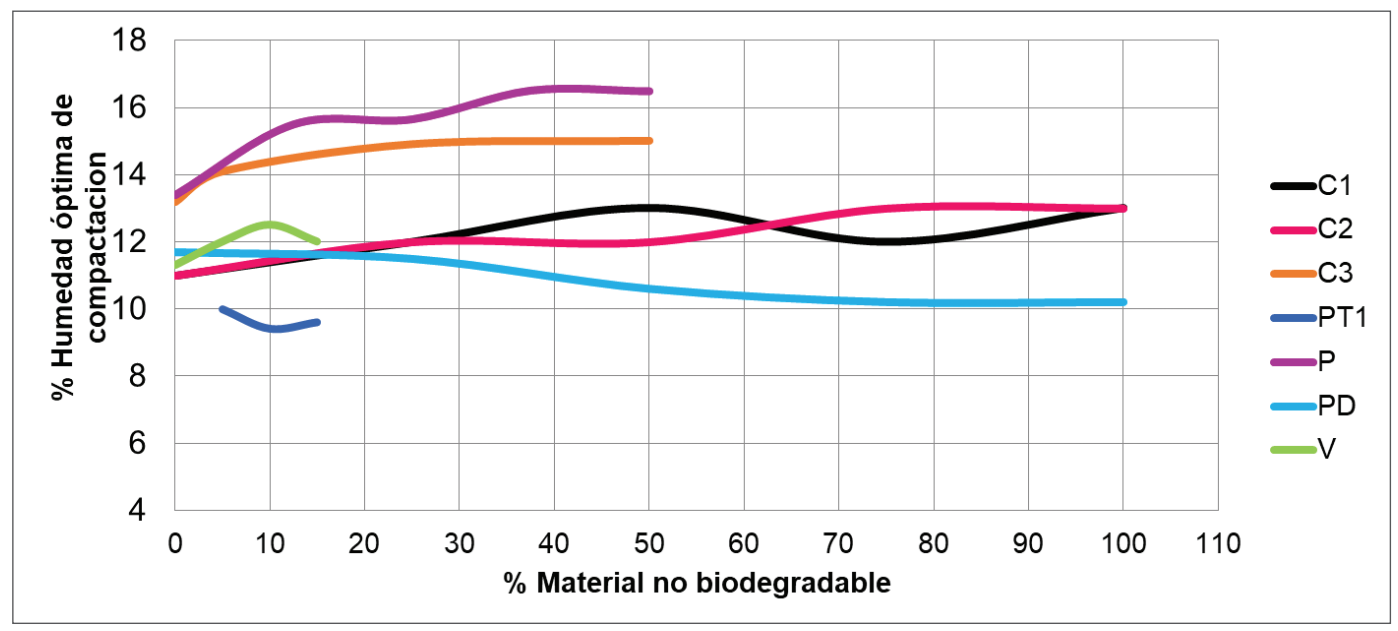

Figura 6. Porcentaje de humedad óptima evaluada en mezclas suelo cemento con material no biodegradable 
En general la variación de la humedad óptima de compactación del suelo natural y de las mezclas modificadas es de aproximadamente $2 \%$, lo cual indica que el material modificador no afecta en gran medida este parámetro, sin embargo, la curva de PT2 no se muestra en la gráfica ya que presenta un comportamiento diferente a las demás, en este caso se siguiere y hace parte de las recomendaciones realizar más pruebas para validar los resultados obtenidos.

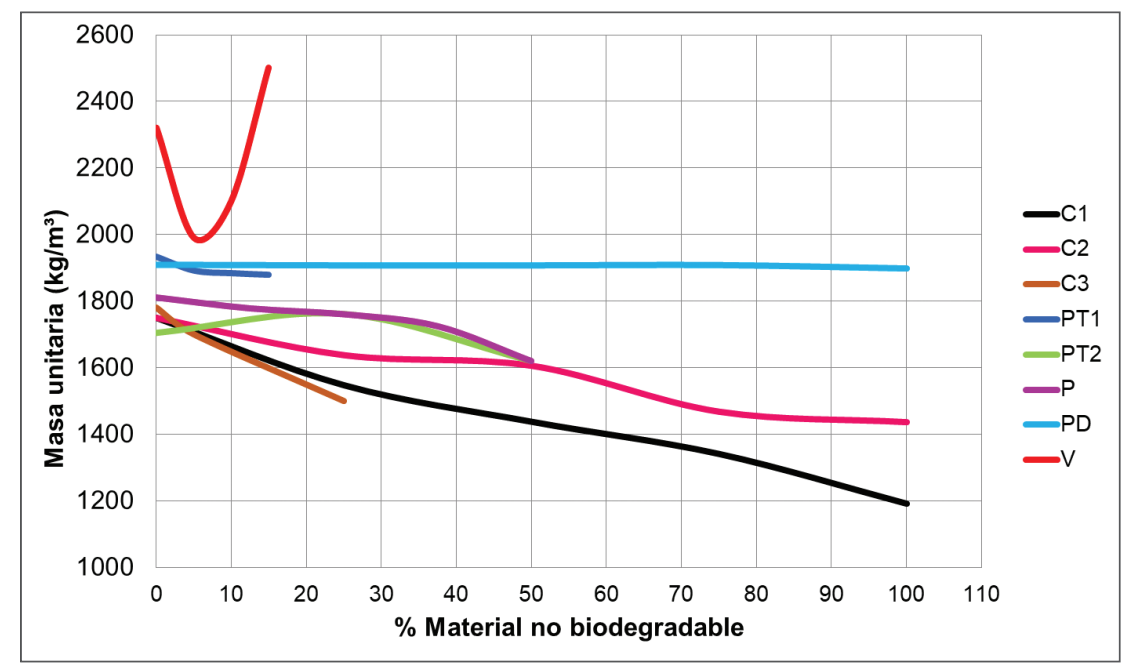

Figura 7. Masa unitaria evaluada en mezclas suelo cemento con material no biodegradable

Adicionalmente la relación entre el material modificador de la mezcla y la masa unitaria es inversamente proporcional, esto es debido a que los materiales modificadores son livianos por lo cual a mayor porcentaje de material modificador en la mezcla menor es su masa unitaria, caso contrario ocurre con el $\mathrm{V}$ ya que su densidad es de $2500 \mathrm{~kg} / \mathrm{m}^{3}$ ocasiona que la masa unitaria de la mezcla presente un incremento.

\section{RESULTADOS}

De la revisión bibliográfica inicial, se plantea el estado de las diferentes condiciones que aún no han sido estudiadas y donde se requiere seguir realizando investigación para validar los resultados respecto a nuevos rangos que se proponen en este artículo. Los cuadros 5,6 y 7 , presentan respectivamente una relación conforme a los materiales y suelos que se han estudiado a la fecha en cuanto a las mezclas suelo cemento modificadas, y por tanto las posibles variaciones e investigaciones que surgen para realizar un estudio más detallado. Los materiales se representan con colores, para su fácil identificación, así:

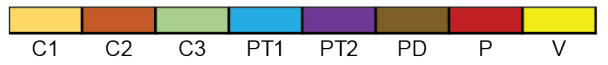

Cuadro 5. Relación de materiales, metodología de modificación y propiedades evaluadas.

\begin{tabular}{|c|c|c|c|c|c|}
\hline \multirow{3}{*}{\multicolumn{2}{|c|}{ Grano de caucho, PET, EPS, Vidrio templado, PEHD }} & \multicolumn{4}{|c|}{ Suelo } \\
\hline & & \multirow{2}{*}{\begin{tabular}{|l|}
$A-1$ \\
$A-1-a$
\end{tabular}} & \multicolumn{2}{|c|}{$\mathrm{A}-2$} & \multirow{2}{*}{$A-4$} \\
\hline & & & A-2-4 & A-2-6 & \\
\hline Método de & Sustitución & & & & \\
\hline modificación & Adición & & & & \\
\hline \multirow{5}{*}{$\begin{array}{c}\text { Propiedades } \\
\text { evaluadas }\end{array}$} & Durabilidad & & & & \\
\hline & Perdidas en peso de material & & & & \\
\hline & Resistencia a la compresión & & & & \\
\hline & Resistencia a la flexión & & & & \\
\hline & Resistencia a la compresión (cubo modificado) & & & & \\
\hline
\end{tabular}


Cuadro 6. Relación de dosificaciones y suelos en las mezclas evaluadas.

\begin{tabular}{|c|c|c|c|c|c|c|}
\hline & \multicolumn{5}{|c|}{ Suelo } & \multirow{2}{*}{ A-4 } \\
\hline \multirow{2}{*}{ \% Dosificación } & A-1 & \multicolumn{3}{|c|}{ A-2 } & \\
\hline & A-1-a & \multicolumn{2}{|c|}{ A-2-4 } & \multicolumn{2}{|c|}{ A-2-6 } & \\
\hline 5 & & & & & & \\
\hline 10 & & & & & & \\
\hline 12.5 & & & & & & \\
\hline 15 & & & & & & \\
\hline 25 & & & & & & \\
\hline 37.5 & & & & & & \\
\hline 50 & & & & & & \\
\hline 75 & & & & & & \\
\hline 100 & & & & & & \\
\hline
\end{tabular}

Cuadro 7. Relación de franjas granulométricas y suelos trabajados en las mezclas evaluadas.

\begin{tabular}{|c|c|c|c|c|}
\hline \multirow{3}{*}{ Tamiz } & \multicolumn{4}{|c|}{ Suelo } \\
\hline & \multirow{2}{*}{$\frac{A-1}{A-1-a}$} & \multicolumn{2}{|c|}{ A-2 } & \multirow{2}{*}{ A-4 } \\
\hline & & A-2-4 & A-2-6 & \\
\hline \multicolumn{5}{|l|}{$3 / 4$} \\
\hline \multicolumn{5}{|l|}{$3 / 8$} \\
\hline \multicolumn{5}{|l|}{ N 4} \\
\hline \multicolumn{5}{|l|}{ N 8} \\
\hline \multicolumn{5}{|l|}{ N 10} \\
\hline \multicolumn{5}{|l|}{ N 16} \\
\hline \multicolumn{5}{|l|}{$\mathrm{N} 20$} \\
\hline \multicolumn{5}{|l|}{ N 30} \\
\hline \multicolumn{5}{|l|}{ N 50} \\
\hline \multicolumn{5}{|l|}{ N 80} \\
\hline \multicolumn{5}{|l|}{ N 100} \\
\hline \multicolumn{5}{|l|}{ N 200} \\
\hline ADICION EN & & & & \\
\hline
\end{tabular}

En los cuadros anteriores todas las casillas en blanco son posibles investigaciones que se pueden realizar a futuro, con el fin de tener una base de datos más amplia. En la Tabla 5 , se presenta la relación del método de modificación y las propiedades evaluadas en las mezclas, donde la PT2 es la mezcla a la que se le realizaron más ensayos; así mismo se presentan en las Tablas 6 y 7, la relación entre los rangos de valores de las dosificaciones y la franja granulométrica con los diferentes suelos, donde estos pueden tener diferentes variaciones y combinaciones.
Partiendo de la comparación realizada, se definen fronteras de cambio con el fin de tener en cuenta la inclusión de estos materiales alternativos en futuras investigaciones, dichas fronteras se proponen en una tabla con rangos de dosificación similares a los establecidos por la PCA (Tabla 8), donde se indica el porcentaje de cemento a adicionar sugerido; la recomendación se hace en función del material modificador y el método de inclusión en la mezcla con el fin de alcanzar la resistencia de 2.1 MPa y con la cual se diseñaron las probetas que se trabajaron en las investigaciones analizadas. 
Cuadro 8. Dosificaciones de diseño sugeridas para la inclusión de materiales no biodegradables.

\begin{tabular}{|c|c|c|c|c|c|}
\hline $\begin{array}{c}\text { Clasificación } \\
\text { AASHTO }\end{array}$ & Clasificación ASTM & $\begin{array}{l}\text { Rango del cemento para } \\
\text { el ensayo de durabilidad } \\
\text { (ASTM D } 559 \text { y D506) \% } \\
\text { por peso según PCA }\end{array}$ & $\begin{array}{l}\text { Material no } \\
\text { biodegradable }\end{array}$ & $\begin{array}{l}\text { Metodología de } \\
\text { inclusión para } \\
\text { material no } \\
\text { biodegradable }\end{array}$ & $\begin{array}{l}\text { Propuesta del rango para } \\
\text { encontrar el contenido de } \\
\text { cemento óptimo con material } \\
\text { no biodegradable }(\%)\end{array}$ \\
\hline A-1-a & GW, GP, GM, SW, SP, SM & $3-5-7$ & PT2 & Sustitución & * \\
\hline \multirow{3}{*}{$A-2-4$} & \multirow{3}{*}{ GM, GP, SM, SC } & \multirow{3}{*}{$5-7-9$} & $\mathrm{C} 1, \mathrm{C} 2$ & Sustitución & $14-27-41$ \\
\hline & & & PT1 & Adición & $3-6-10$ \\
\hline & & & V & Adición & * \\
\hline \multirow{2}{*}{ A-2-6 } & \multirow{2}{*}{$\mathrm{GM}, \mathrm{GP}, \mathrm{SM}, \mathrm{SC}$} & \multirow{2}{*}{$5-7-9$} & PD & Sustitución & $13-28-43$ \\
\hline & & & $\mathrm{P}$ & Sustitución & $30-33-36$ \\
\hline$A-4$ & $\mathrm{CL}, \mathrm{ML}$ & 8-10-12 & $\mathrm{C3}$ & Adición & $13-17-21$ \\
\hline
\end{tabular}

Nota: * No se propone nada porque la resistencia mínima a la compresión se logra dentro del rango de la PCA.

\section{CONCLUSIONES}

En la Tabla 8, de los 5 tipos de materiales no biodegradables que modificaron las mezclas, fue posible lograr la resistencia mínima requerida por el artículo 350 del Invías, dentro del rango de cemento sugerido por la PCA para el PET y el vidrio. En el caso del PET, la validación de la PCA, se da para los dos métodos de modificación (adición o sustitución).

Para el grano de caucho por método de modificación de sustitución, el nuevo rango sugerido en los porcentajes de cemento para la fabricación de las probetas, es muy diferente al planteado por la PCA. En el caso del mismo modificador por el método de adición, el nuevo rango también es diferente al de la PCA, pero no tan distante; esto puede implicar que para una mezcla modificada por un material liviano como el PEHD la metodología no se ajusta.
Referente a lo anterior, para los modificadores EPS y PEHD, se puede concluir lo mismo, por tratarse de materiales de muy baja densidad, la resistencia a la compresión de 2.1 MPa no se logra para los porcentajes de cemento recomendados, al igual que la máxima pérdida permitida por efecto de la evaluación de durabilidad por la prueba de humedecimiento y secado, por lo que se proponen unos nuevos rangos de cemento óptimo. De acuerdo con esto se concluye que las bajas densidades de materiales alternativos, ocasionan que la metodología PCA para diseño de mezclas suelo cemento modificadas no se ajuste.

Dada la cantidad de datos utilizados en este documento de acuerdo con las tablas presentadas del estado del conocimiento actual para las mezclas suelo cemento modificadas, es necesario seguir ampliando la investigación de manera que a futuro se pueda proponer una metodología similar a la PCA con nuevos valores y que estos estén validados con suficiencia estadística.

\section{REFERENCIAS BIBLIOGRÁFICAS}

1. Acero, A. Y Mogollón, F. (2015). Análisis de una muestra suelo- cemento sustituyendo la fracción granulométrica (pasa no 8 - retiene no 16) por grano de caucho. Bogotá: Universidad de La Salle.

2. Asociación nacional de poliestireno expandido (2017). Madrid. Recuperado de https://goo.gl/mzkVQy

3. Buitrago, H. y González, E. (2015). Comportamiento de una base estabilizada con cemento sustituyendo partículas de poliestireno expandido en la fracción fina del agregado (tamiz \# 10 y \# 20). Bogotá: Universidad de La Salle.

4. Capas de material granular estabilizado con cemento sección 420-11, Instituto de desarrollo urbano IDU, Bogotá D.C. 2011. Recuperado de https://goo.gl/ ElnUiH

5. Características del PET, M\&M Plásticos. 2012. Recuperado de https://goo.gl/xjc733

6. Colombia apuesta por el reciclaje de vidrio. 2012 Recuperado de https://goo.gl/MegPIF.

7. Colombia entierra millones de pesos por no reciclar, Rojas F. Publicado el 10 de enero de 2016, Periódico El Colombiano. https://goo.gl/nXQ5nW.

8. Cruz, J. y Díaz, I. (2015). Utilización de caucho pulverizado proveniente de llantas en bases estabilizadas con suelo - cemento sustituyendo la franja granulométrica entre el tamiz n³0 y n80. Bogotá: Universidad de La Salle.

9. Degradación del plástico. Recuperado de https://goo.gl/mIOuOw 
10. Factibilidad económica, ambiental y social del proceso de fabricación de adoquines cpm de flexiform como alternativa para la formación de recuperadores de Bogotá D.C. Álzate S. y Tafur F. Universidad de La Salle, Bogotá. 2006.

11. Ikoportex- poliestireno expandido. Recuperado de https://goo.gl/1FddVp

12. Infante S. (2015). Evaluación de características físicas y mecánicas de una base estabilizada con cemento modificada con vidrio templado. Bogotá: Universidad de La Salle.

13. Inforeciclaje - Reciclaje del vidrio. Recuperado de https://goo.gl/JPp96x

14. Invías (2013), Especificaciones Generales de Construcción de Carreteras y Normas de Ensayo para Materiales de Carreteras, Instituto Nacional de vías, Colombia.

15. Junca. I (2014). El icopor, una amenaza ambiental creciente para Bogotá. Periódico El Espectador. Bogotá. Recuperado de https://goo.gl/OTPgHP

16. La oportunidad está en la basura. Revista Dinero, 2009. Recuperado de https://goo.gl/BMMPVE

17. Meneses L. y Fuentes L. (2015). Base estabilizada con cemento, modificada con PET reciclado. Bogotá: Universidad de La Salle.

18. Montejo, A. (2006). Ingeniería de pavimentos (Vol. 2). Bogotá: Universidad Católica de Colombia.

19. Ochoa, P. y Valderrama, C. (2012). Base estabilizada con cemento modificada con grano de caucho de llanta. Bogotá: Universidad de La Salle.

20. Propiedades generales del vidrio. Recuperado de: https://goo.gl/BzpJiC

21. Ramírez. N (2006). Estudio de la utilización de caucho de neumáticos en mezclas asfálticas en caliente mediante proceso seco. Universidad de chile. Santiago de chile.

22. Resolución 1457 de 2010, Ministerio de Ambiente - Colombia. Recuperado de https://goo.gl/DEDATU

23. Rico, A. y Del Castillo, E. (2009). La ingeniería de los suelos en las vías terrestres (Vol. 2). Limusa editores.

24. Sarmiento J. y Bustos D. (2016). Análisis del comportamiento de base granular estabilizada con cemento sustituyendo con material no biodegradable tereftalato de polietileno (PET) en la fracción del agregado que pasa el tamiz \#4 y se retiene en el \#16. Bogotá: Universidad de La Salle.

25. Sector plástico, guías ambientales. 2004. Ministerio de Ambiente, Vivienda y Desarrollo Territorial, Republica de Colombia. Bogotá.

26. State of the Art Report in Soil Cement, ACI 230.1R - 90, 1997. Recuperado de https://goo.gl/hBV9AT

27. Suelo - cemento 1ra parte. Instituto Mexicano del Cemento y el Concreto A.C. Ingeniería, marzo 2008. Recuperado de https://goo.gl/X2ZKdc

28. Suelo - cemento 2da parte. Instituto Mexicano del Cemento y el Concreto A.C. Ingeniería, abril 2008. Recuperado de https://goo.gl/7RDkdP

29. Velandia, C y Parra, L. (2016). Suelo A-2,6 estabilizado con cemento sustituyendo la fracción pasa el tamiz nº 4 y retiene $n^{\circ} 10$ del agregado por polímero de alta densidad (PE-HD), Bogotá: Universidad de La Salle. 